\title{
Comparison between minimally invasive plate osteosynthesis and the deltopectoral approach with allogenous fibular bone graft in proximal humeral fractures
}

\author{
Joon Yub Kim ${ }^{1}$, Jinho Lee ${ }^{2}$, Seong-Hun Kim ${ }^{2}$ \\ ${ }^{1}$ Department of Orthopaedic Surgery, Yeson Hospital, Bucheon, Korea \\ ${ }^{2}$ Department of Orthopedic Surgery, National Health Insurance Service Ilsan Hospital, Goyang, Korea
}

\begin{abstract}
Background: The purpose of this study was to investigate the clinical differences between open reduction and plate fixation via a deltopectoral approach with allogenous fibular bone graft and a minimally invasive plate osteosynthesis (MIPO), in Neer's classification two-, threepart proximal humeral fractures.

Methods: In this retrospective study, 77 patients with two-, three-part proximal humeral fractures were treated at two different institutions. Clinical and radiological evaluations were performed in 39 patients, who underwent MIPO at one institution (group A), and 38 patients, who underwent a deltopectoral approach with allo-fibular bone graft (group B) at another institution. The results between the groups were compared.

Results: The MIPO technique was significantly less time consuming and caused less bleeding than the deltopectoral approach with allo-fibular bone graft $(\mathrm{P}<0.05)$. The duration of the fracture union was significantly reduced in group A $(14.5 \pm 3.4$; range, $10-22$ weeks $)$ compared to group B $(16.4 \pm 4.3$; range, $12-28)$ weeks $(\mathrm{P}<0.05)$. There were no statistically significant differences between the two groups when evaluating the visual analog scale and Constant scores between the two groups, 1 year postoperatively. In radiological evaluation, there was no difference in radiological outcomes between the two groups. There were no statistically significant differences in malunion between the two groups.

Conclusions: The MIPO technique and deltopectoral approach with allo-fibular bone graft for two-, three-part proximal humeral fractures, show similar clinical and radiological results. However, allogenous fibular grafts require longer surgery, cause more bleeding, and result in longer fracture healing time than MIPO technique.
\end{abstract}

Keywords: Proximal humeral fractures; MIPO; Delto-pectoral approach; Fibular allograft

\section{INTRODUCTION}

Proximal humeral fractures are relatively common fractures, accounting for about $5 \%$ of all fractures and are more frequent in older osteoporosis patients [1]. In most cases without severe displacement, good outcomes can be achieved through non-surgical treatment [2]. However, in cases in which bone fragments have large displacement or angulation, surgical treatment is required

Received: July 31, $2020 \quad$ Revised: August 12, $2020 \quad$ Accepted: August 15, 2020

Correspondence to: Seong-Hun Kim

Department of Orthopedic Surgery, National Health Insurance Service Ilsan Hospital, 100 Ilsan-ro, Ilsandong-gu, Goyang 10444, Korea

Tel: +82-31-900-0436, Fax: +82-31-900-0343, E mail: esmeron@nhimc.or.kr, ORCID: https://orcid.org/0000-0003-1831-7930

Financial support: None.

Conflict of interest: None.

Copyright@ 2020 Korean Shoulder and Elbow Society. All Rights Reserved.

This is an Open Access article distributed under the terms of the Creative Commons Attribution Non-Commercial License (http://creativecommons.org/licenses/by-nc/4.0/) which permits unrestricted non-commercial use, distribution, and reproduction in any medium, provided the original work is properly cited. 
to restore normal shoulder function [3]. Surgical treatment methods include plate fixation, intramedullary nailing, and replacement arthroplasty; however, internal fixation with a locking plate is the most commonly used treatment method [4]. Patients with severe comminuted fractures or osteoporosis are more likely to develop complications such as fixation failure, interposition, and angulation during normal plate fixation. These complications often lead to poor clinical outcomes. Recently, allogenous fibular bone graft with relatively high bone strength and similar size to the inner diameter of the proximal humerus has been used to prevent these problems. Furthermore, recent studies have shown that in cases of severe comminuted fractures, superior clinical results were achieved using the graft method [5-7].

The deltopectoral approach was previously used for plate fixation in proximal humeral fractures. However, this approach requires a large incision range and considerable dissection of the soft tissue to secure the field of view of the lateral part of the humerus [8]. In order to address these drawbacks, plate fixation with minimally invasive plate osteosynthesis (MIPO) can shorten the surgery time and minimize bleeding and soft tissue injury, leading to superior fracture healing compared to the conventional deltopectoral approach $[8,9]$. In some studies, fixation with MIPO has demonstrated superior clinical outcomes compared to open reduction $[8,10]$. However, due to the limited visibility during surgery, especially in Neer classification three-, four-part with severe displacement, skillful operation is required for proper reduction. Due to the limited approach, performance of bone grafts including allogenous fibular bone grafts is difficult, especially when there is no greater tuberosity fracture or the extent of displacement is not severe [11].

Both allogenous fibular bone graft and MIPO have been introduced to minimize the problems of fixation failure, displacement, angulation, and nonunion after plate fixation of proximal humeral fractures. However, there have been no studies investigating the differences between the clinical results of these two treatment methods. The aim of this study was to investigate the clinical differences between plate fixation via the deltopectoral approach with allogenous fibular bone graft and MIPO without bone graft in proximal humeral fractures.

\section{METHODS}

This study was approved by the Institutional Review Board of National Health Insurance Service Ilsan Hospital (IRB No. 201704-036).

\section{Patient Selection}

This study is a retrospective analysis of the medical records of patients diagnosed with proximal humeral fractures who underwent internal fixation with locking plates by the same shoulder surgeon with similar clinical experiences in two different medical institutions from January 2014 to December 2016. Patients over 19 years old with two-, three-part proximal humeral fracture who underwent surgical treatment were included. Patients under 19 years of age, with associated clavicle or scapula fractures, neurological injuries such as brachial plexus injury, periprosthetic fracture, and those without at least 1 year of follow-up were excluded from the study. One surgeon performed locking plate fixation using MIPO in all patients with Neer classification two-, three-part fractures except for cases of greater tuberosity fractures alone. The other surgeon at another institution performed locking plate fixation and allogenous fibular bone graft via the deltopectoral approach in all two-, three-part fracture patients. Cases of four-part fractures were excluded from the analysis because MIPO was not performed in any. A total of 77 patients met the inclusion criteria. Clinical and radiological results were analyzed and compared for 39 patients who underwent MIPO (group A) and 38 patients who underwent allogenous fibular bone graft (group B).

\section{Operative Technique}

All patients underwent surgery in the beach chair position and the surgery was performed using image amplification equipment (C-arm) monitoring the status of reduction. Surgeries for both groups utilized the same locking plate type (PHILOS; Synthes, Paoli, PA, USA).

For MIPO, a 3-cm skin incision was made from the anterolateral side of the acromion to the distal part. The anterior raphe of the deltoid was detached and a finger was placed to feel the axillary nerve under the deltoid of the distal incision line. This was lifted to protect the nerve. Under the deltoid on the lateral side of the periosteum, blunt dissection was performed to create space to insert the plate. Greater tuberosity fracture with displacement cases were reduced while monitoring with image amplification equipment and fixed with two Kirschner wires. Upon securing enough space to place the plate under the deltoid and along the humeral surface, the axillary nerve was lifted and protected using a finger and the plate was inserted into the space. While monitoring using image amplification equipment, the plate was placed at an estimated height of $5 \mathrm{~mm}$ distal to the greater tuberosity. With an oblong-shaped hole in the distal plate as a reference, an approximate $3-\mathrm{cm}$ incision was made in the distal part. Intramuscular dissection was performed to expose the distal plate. For 
indirect reduction, a pin was inserted into the anterior humeral head, and the reduction was performed using the joystick method. Alternatively, a conventional screw was inserted in the oblong-shaped hole in the distal plate for indirect humeral head reduction. After investigating the state of reduction with the image amplification equipment, the humeral head was fixed by six locking screws from the proximal plate to the third row. The arm was used to secure adequate visualization of the distal plate, and the remaining locking screws were inserted to complete the procedure. Since the medial inferior screws of the fourth row of the plate were placed near the axillary nerve, these were not inserted in all patients (Fig. 1).

In the allogenous fibular bone graft group, the deltopectoral approach was performed in all patients. Commencing at the coracoid process end, an $\sim 10 \mathrm{~cm}$ incision was made in the lateral inferior direction toward the deltoid attachment. The subcutaneous tissue was dissected with the cephalic vein placed to the lateral side, and a blunt dissection was performed between the deltoid and the pectoralis major muscle. The fractured proximal humerus was exposed by dissection of the attachment site under the deltoid and removal of the bursa tissue. After the allogenous fibula bone was trimmed to enter the humerus inner diameter, the fracture site was opened to secure a space. The allogenous fibula bone was inserted while monitoring with the image ampli- fication equipment, resulting in indirect reduction of the humerus fracture site by allogenous fibula bone. In cases with greater tuberosity fractures, a few threads were passed through the supraspinatus tendon and infraspinatus tendon, applying traction to the fragments for reduction; temporary fixation was performed using K-wires. Complete reduction state of the fracture site was confirmed with the image amplification equipment, and the plate was secured by fixing the screw to complete the surgery (Fig. 2).

All patients were immobilized for 3 to 6 weeks using a sling after surgery. After pain was mitigated, the patients began passive exercise and assisted active exercise on a set schedule. Muscle strength exercise was performed after fracture union.

\section{Clinical and Radiological Assessments}

For clinical evaluation, the amount of bleeding during surgery was measured by referring to the medical record prepared by the anesthesiologist, and the surgery time was defined as the time from the first skin incision to completed suture. The visual analog scale (VAS) and Constant score were measured 1 year after surgery for functional evaluation. After the surgery, all patients were treated as outpatients, visiting the hospital on a regular basis. The anteroposterior (AP) X-ray and axillary X-ray images were examined to determine the reduction and maintenance sta-
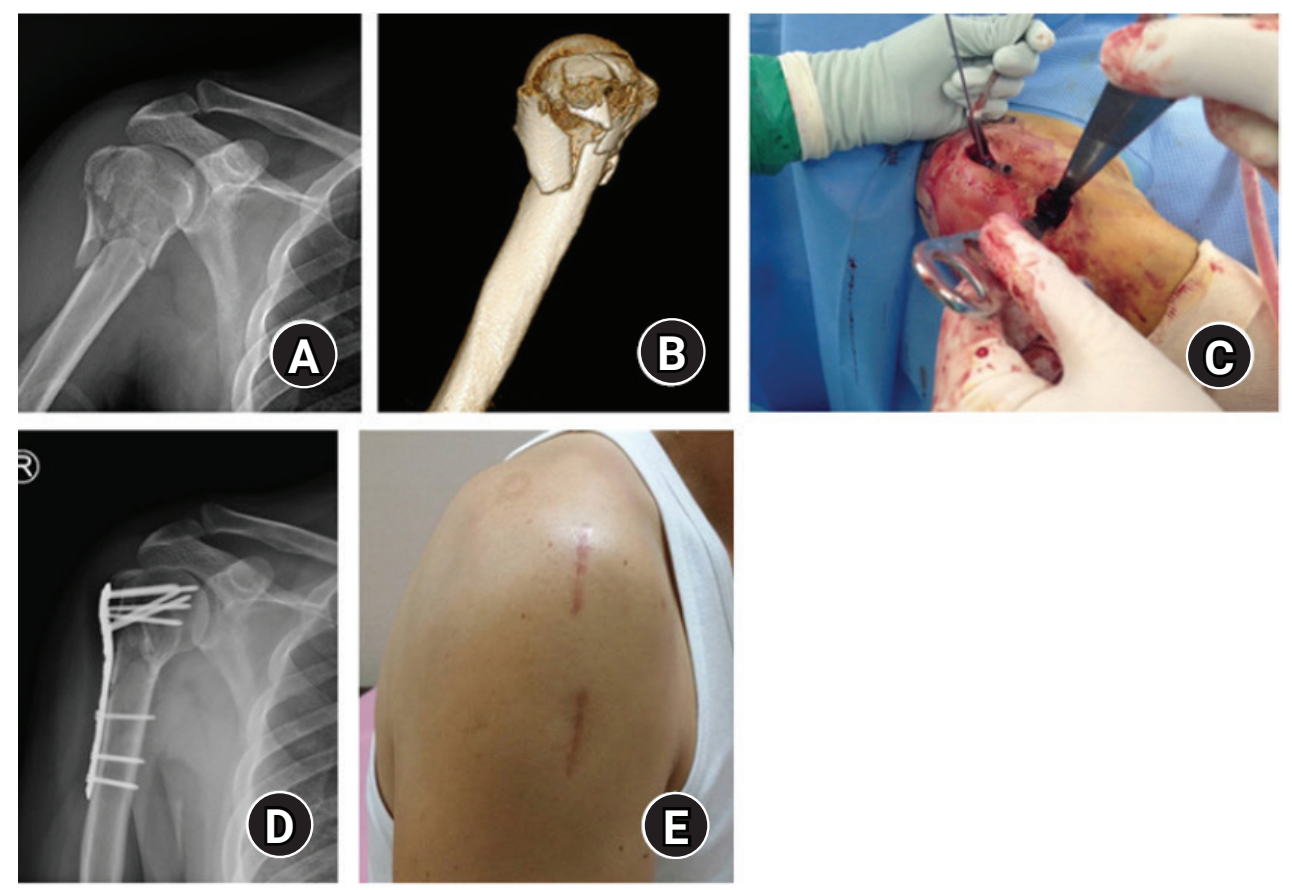

Fig. 1. A 50-year old man with comminuted proximal humeral fracture who was treated with minimally invasive plate osteosynthesis technique. (A) Preoperative anteroposterior radiography. (B) Preoperative three-dimensional computed tomography scan. (C) Intraoperative gross photo. (D) Postoperative anteroposterior radiography. (E) Final scar from surgery. 

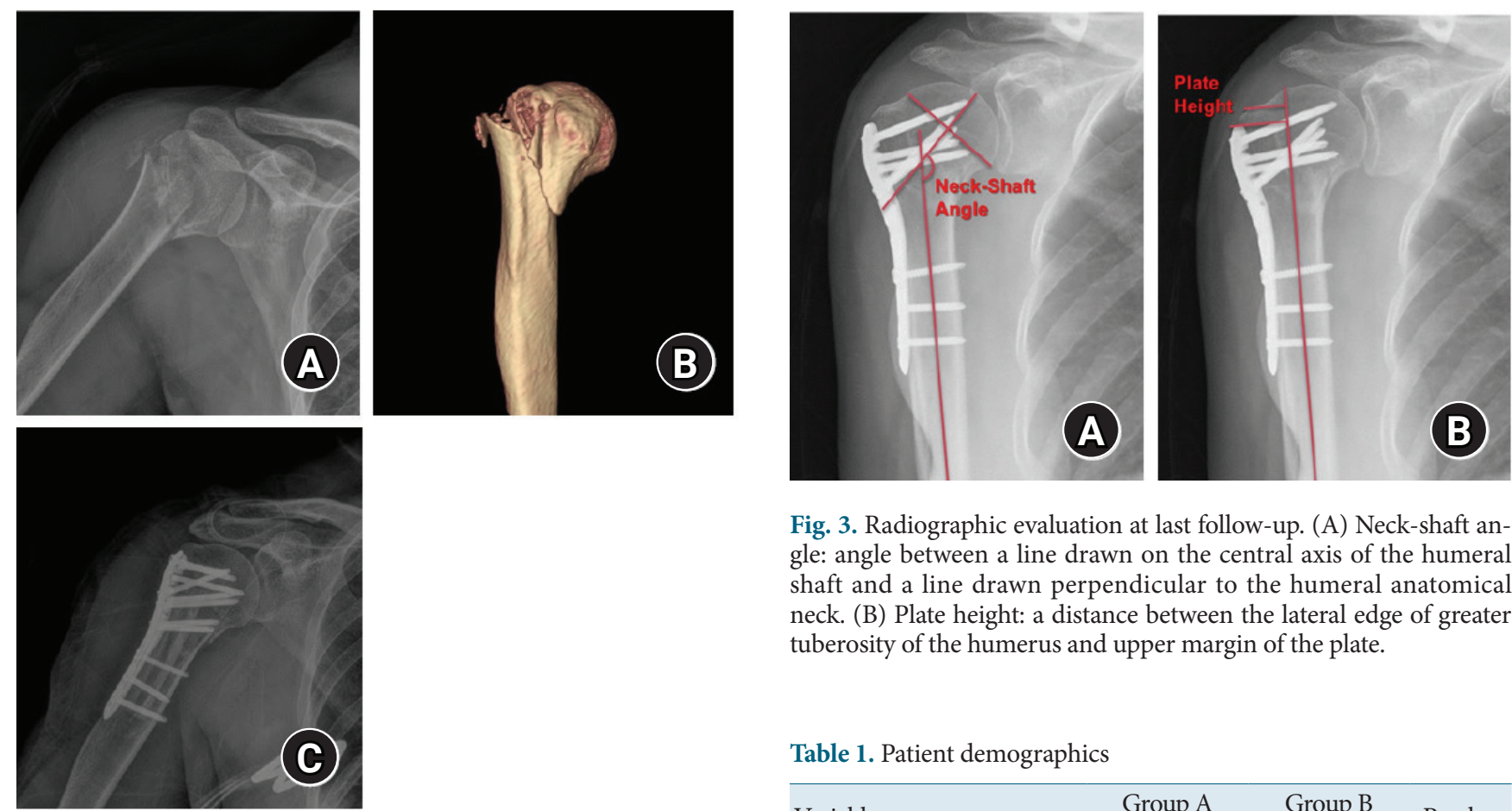

Fig. 3. Radiographic evaluation at last follow-up. (A) Neck-shaft angle: angle between a line drawn on the central axis of the humeral shaft and a line drawn perpendicular to the humeral anatomical neck. (B) Plate height: a distance between the lateral edge of greater tuberosity of the humerus and upper margin of the plate.

Table 1. Patient demographics

\begin{tabular}{lccc}
\hline Variable & $\begin{array}{c}\text { Group A } \\
(\mathrm{n}=39)\end{array}$ & $\begin{array}{c}\text { Group B } \\
(\mathrm{n}=38)\end{array}$ & P-value \\
\hline Age (yr) & $68.1 \pm 12.7$ & $69.8 \pm 14.5$ & 0.582 \\
Sex (male:female) & $7: 32$ & $5: 33$ & 0.562 \\
Preoperative BMD, T score & $-2.92 \pm 0.83$ & $-2.86 \pm 0.91$ & 0.754 \\
Neer classification* $(2: 3)$ & $20: 19$ & $24: 14$ & 0.292 \\
Follow-up period (mo) & $15.9 \pm 5.6$ & $17.2 \pm 6.4$ & 0.348 \\
\hline
\end{tabular}

Values are presented as mean \pm standard deviation. Group A: minimally invasive plate osteosynthesis, Group B: deltopectoral approach with allogenous fibular bone graft.

BMD: bone mineral density.

${ }^{*}$ Neer classification: proximal humeral fractures classification based on the number of displaced parts, two-part and three-part fractures.

least three bridging calluses observed in the AP and axillary $\mathrm{X}$-ray images. For radiological evaluation, neck-shaft angle was measured on the final follow-up AP images (Fig. 3A) [12]. Malunion was defined as a neck-shaft angle less than $120^{\circ}$ on postoperative radiographs during the follow-up period [11]. Plate height was measured as the distance from the lateral end of the humeral greater tuberosity to the upper end of the plate in the final follow-up AP image (Fig. 3B).

\section{RESULTS}

The group that underwent plate fixation using MIPO (group A) consisted of 39 patients; and 38 patients were in group $B$, the group that underwent plate fixation using the deltopectoral approach with allogenous fibular bone graft. Group A had 20 cases of Neer classification two-part fractures (51.3\%) and 19 cases of three-part fractures (48.7\%); group B had 24 cases of two-part fractures $(63.2 \%)$ and 14 cases of three-part fractures (36.8\%). There were no statistical differences in the basic characteristics between the two groups prior to surgery (Table 1).

The surgery time was significantly shorter in group A $(73.4 \pm 17.9$ minutes) than in group $\mathrm{B}(146.2 \pm 53.7$ minutes $)$ patients $(\mathrm{P}<0.001)$. Intraoperative bleeding was also significantly lower in group $\mathrm{A}$ $(86.2 \pm 65.4 \mathrm{~mL})$ than in group $\mathrm{B}(279.6 \pm 189.2 \mathrm{~mL} ; \mathrm{P}<0.001)$. There was no statistically significant difference between the two groups in the evaluation of clinical function by VAS score and Constant score at the 1-year postoperative follow-up (Table 2).

Postoperative radiological evaluation showed fracture union in both groups, and no nonunion was observed. The mean time for fracture union after surgery was $14.5 \pm 3.4$ weeks (range, 10-22 weeks) in group A patients and $16.4 \pm 4.3$ weeks (range, 12-28 weeks) in group B patients. Fracture union time was statistically significantly shorter for the group A patients $(P=0.032)$. The neck-shaft angle measured on the AP X-ray images after surgery was $130.4^{\circ} \pm 6.5^{\circ}$ (range, $117.8^{\circ}-141.1^{\circ}$ ) in group $\mathrm{A}$ and $132.9^{\circ} \pm 8.7^{\circ}$ 
Table 2. Clinical outcomes

\begin{tabular}{lccc}
\hline Variable & Group A & Group B & P-value \\
\hline VAS & $1.3 \pm 1.1$ & $1.4 \pm 1.0$ & 0.636 \\
Constant score $^{\dagger}$ & $81.8 \pm 9.1$ & $78.3 \pm 7.5$ & 0.067 \\
Operation time $(\mathrm{min})$ & $73.4 \pm 17.9$ & $146.2 \pm 53.7$ & $<0.001^{\star}$ \\
Intraoperative bleeding $(\mathrm{mL})$ & $86.2 \pm 65.4$ & $279.6 \pm 189.2$ & $<0.001^{\star}$ \\
\hline
\end{tabular}

Values are presented as mean \pm standard deviation. Group A: minimally invasive plate osteosynthesis, Group B: deltopectoral approach with allogenous fibular bone graft.

VAS: visual analog scale.

${ }^{*}$ Statistically significant difference between the two groups $(\mathrm{P}<0.05)$;

${ }^{\dagger}$ Constant score evaluated on 1-year follow up.

Table 3. Radiographic outcomes in both groups

\begin{tabular}{lccc}
\hline Variable & Group A & Group B & P-value \\
\hline Time to union $(\mathrm{wk})$ & $14.5 \pm 3.4$ & $16.4 \pm 4.3$ & $0.032^{\star}$ \\
Neck-Shaft angle $^{\dagger}\left({ }^{\circ}\right)$ & $130.4 \pm 6.5$ & $132.9 \pm 8.7$ & 0.163 \\
${\text { Plate height }(\mathrm{mm})^{\ddagger}}^{\ddagger}$ & $8.1 \pm 2.1$ & $8.8 \pm 3.3$ & 0.250 \\
Malunion $^{\S}$ & 3 & 4 & 0.665 \\
\hline
\end{tabular}

Values are presented as mean \pm standard deviation. Group A: minimally invasive plate osteosynthesis, Group B: deltopectoral approach with allogenous fibular bone graft.

*Statistically significant difference between the two groups $(\mathrm{P}<0.05)$;

${ }^{\dagger}$ Angle between a line drawn on the central axis of the humeral shaft and a line drawn perpendicular to the humeral anatomical neck; ${ }^{\ddagger} \mathrm{A}$ distance between the lateral edge of greater tuberosity of the humerus and upper margin of the plate; ${ }^{8}$ Defined as an angle of $<120^{\circ}$ or $>145^{\circ}$ at final follow-up anteroposterior view.

(range, $112.7^{\circ}-155.1^{\circ}$ ) in group $\mathrm{B}$, indicating no difference between the two groups. The plate height was $8.1 \pm 2.1 \mathrm{~mm}$ (range, $3.6-13.4 \mathrm{~mm}$ ) in group A and $8.8 \pm 3.3 \mathrm{~mm}$ (range, 3.0-15.1 mm) in group $\mathrm{B}$, indicating no difference between the two groups. At the radiological evaluation of the final follow-up, the occurrence of malunion (3 cases [7.7\%] in group A and 4 cases [10.5\%] in group B) (Table 3) did not show a statistically significant difference between the two groups.

\section{DISCUSSION}

There have been a number of comparative studies on the clinical and radiological outcomes of different approaches in the surgical treatment of proximal humeral fractures [8,13-18]. Conventionally, the deltopectoral approach is the most commonly used approach for the proximal humerus. This approach is performed in the anterior direction but has the disadvantage of difficulty in maneuvering the fragments in the greater tuberosity area. Also, since the plate is positioned on the lateral side of the humerus, visibility is limited [11]. An extended anterolateral deltoid-splitting approach can be performed; however, there is a possibility of axillary nerve injury with this approach [19]. Hepp et al. [13] re- ported that this approach had better clinical outcomes compared to the deltopectoral approach due to the deltoid injury occurring in the operation process. In comparison to the deltopectoral approach, MIPO has been introduced and widely used because this method minimizes deltoid injury [20] and facilitates the placement of the plate. This method also ensures biological healing without compromising the blood flow at the periosteum [21]. There have been various reports on differences in clinical and radiological results between the MIPO and deltopectoral approaches. In particular, the recent meta-analysis by Li et al. [17] showed that MIPO had better outcomes than open reduction in terms of bleeding during surgery, operation time, postoperative pain, time taken for bone union, and functional score. However, this method required a longer radiation exposure time during the surgery and had a higher incidence of axillary nerve injury. Sohn et al. [15] reported no significant differences in the clinical and radiological evaluation of the two approaches in prospective studies, but MIPO was significantly less time-consuming. This can be advantageous in the treatment of polytrauma patients. In this study, allogenous fibular bone graft, which is not a simple open reduction, was performed in all patients; as a result, similar to previous findings, MIPO involved less bleeding during surgery and less surgical time. This was significantly different compared to the allogenous fibular bone graft group. Additionally, the time required for the fracture healing was less in the MIPO because, in order to perform allogenous fibular bone graft, spreading the fracture site for space is necessary. With MIPO, however, periosteum is kept intact. This is advantageous in terms of fracture healing. In this study, no axillary nerve injury was found among the patients who underwent MIPO. Axillary nerve injury can be prevented during the surgery by prior palpation of the area in which the axillary nerve passes under the deltoid and protecting the nerve using a finger. The nerve is also protected by performing blunt dissection using Cobb retractor, by use of the opposite finger when inserting a plate, and by inserting the plate with deltoid lifted.

Attempts have been made in order to prevent problems such as cutout or loosening of the screw or varus malalignment that may occur after the plate fixation procedure in patients with severe osteoporosis or comminuted fracture. One method used has been the intramedullary allogenic bone graft [22-24]. In addition, several studies compared clinical and radiological results with and without allogenous bone graft in patients who underwent open reduction using the same locking plate. Cha et al. [25] reported that, in radiological evaluation in the postoperative follow-up, allogenous bone graft maintained the reduction better. Furthermore, Cui et al. [26] also recently reported that in terms 
of clinical results, the allogenous bone graft group had better outcomes. In this study, however, there was no difference in clinical and radiological results between the patients who underwent plate fixation through MIPO and those who underwent allogenous fibular bone graft in open reduction in the final postoperative follow-up. There was also no difference in the number of malunion occurrences.

Considering that the bone densities measured in the two groups were similar and that the fracture patterns of the two groups evaluated by Neer classification were also similar, the findings indicate that the neck-shaft angle is maintained after surgery to the same extent as providing sufficient medial support with a bone graft although there was no medial calcar screw insertion in the MIPO. In the case of MIPO with accompanying medial comminution, the immobilization period was longer than that of other patients and rehabilitation was performed slowly. The results indicate that with the open reduction, excess intervention on the fracture site is inevitable during the allogenous fibula bone graft process through open reduction, which certainly causes injury to the periosteum and in the soft tissue. Such intervention of the fracture site can be minimized in the simple MIPO, and, barring the presence of a severe comminuted fracture, sufficient support of the fracture site can be provided.

Neer classification four-part fractures were not included in this study. In the case of four-part fractures, difficulty arises in precise reduction of the fracture site through MIPO. In particular, there are cases when allogenous fibular bone graft is needed, such as severe comminuted fracture of the medial cortical bone, but treatment by MIPO has limitations. Recently, Noh et al. [27] reported that allogenous fibular bone grafts with MIPO for three-, four-part fractures showed good clinical results. Sohn and Shin [11] examined the difference in clinical and radiological results according to the type of fracture in the group of patients with proximal humeral fractures who underwent MIPO. This group reported that difficult arises in achieving good outcomes with MIPO in four-part fractures. Although not included in this study, the authors attempted MIPO in the case of a four-part fracture; but sufficient reduction was not possible, so the surgery proceeded with an extended anterolateral deltoid-splitting approach. The most important aspect in the internal fixation of fractures is the sufficient anatomical reduction of the fracture site rather than the surgical approach. Therefore, the flexibility is required in choosing MIPO or open reduction for sufficient reduction of the fracture site.

In this study, the two surgeons performed surgical treatment on the same group of patients only with their respective preferred surgical method. One surgeon performed the surgery with a belief that MIPO alone could provide sufficient fixation for twoand three-part fractures. Another surgeon preferred open reduction using allogenous fibular bone graft in all patients with twoor three-part fracture to facilitate reduction. This allowed for support of the medial cortex of inserted bone by use of an intramedullary nail. Furthermore, the two medical institutions are adjacent to each other, and the patient groups are similar. The two surgeons have similar clinical and surgical experiences, and the general characteristics of the two patient groups did not differ. Under these conditions, we believe that this study was suitable to compare between the two surgical methods.

Based on the results of this study, performing allogenous fibular bone graft in two- or three-part fractures does not seem to have a significant advantage over performing MIPO alone. Rather, allogenous fibular bone grafting caused longer surgery times, increased bleeding, and prolonged fracture healing. In particular, considering the additional cost from the use of allogeneic bone and risk of complications such as infection, we believe that the concurrent allogenous fibular bone graft does not have advantages for general use. Kim et al. [28] also reported that the performance of allogenous fibular bone graft did not significantly affect the clinical outcomes in three-part fractures.

This study had some limitations. First, the population size was relatively small. Also, this was a retrospective study, and different surgeries were performed by two different surgeons in two medical institutions. Although both practitioners are shoulder surgeons with similar clinical experience, ruling out the possibility of subjective intervention by the practitioner in terms of classifying fractures and determining the need for surgical method is difficult. However, considering that the surgery was performed using the same instrument and there were no complications such as malunion after surgery, we believe that the difference between the surgeons did not have a significant impact on the results. The importance of this study is that this is the first study to compare the differences between plate fixation through MIPO and allogenous fibular bone graft through open reduction.

The MIPO and allogenous fibular bone graft through open reduction showed similar clinical and radiological results in the fixation using locking plate in Neer classification two- or threepart proximal humeral fractures. However, allogenous fibular bone grafts required longer surgical time, caused more bleeding, and required a longer time for fracture healing compared to MIPO. Hence, careful consideration is required when selecting the fixation method according to fracture comminution. 


\section{ORCID}

\section{Joon Yub Kim \\ Jinho Lee \\ Seong-Hun Kim}

miology of proximal humeral fractures. Clin Orthop Relat Res 2006;442:87-92.

13. Hepp P, Theopold J, Voigt C, Engel T, Josten C, Lill H. The surgical approach for locking plate osteosynthesis of displaced proximal humeral fractures influences the functional outcome. J Shoulder Elbow Surg 2008;17:21-8.

\section{REFERENCES}

1. Horak J, Nilsson BE. Epidemiology of fracture of the upper end of the humerus. Clin Orthop Relat Res 1975;(112):250-3.

2. Gaebler C, McQueen MM, Court-Brown CM. Minimally displaced proximal humeral fractures: epidemiology and outcome in 507 cases. Acta Orthop Scand 2003;74:580-5.

3. Pinkas D, Wanich TS, DePalma AA, Gruson KI. Management of malunion of the proximal humerus: current concepts. J Am Acad Orthop Surg 2014;22:491-502.

4. Hirschmann MT, Fallegger B, Amsler F, Regazzoni P, Gross T. Clinical longer-term results after internal fixation of proximal humerus fractures with a locking compression plate (PHILOS). J Orthop Trauma 2011;25:286-93.

5. Panchal K, Jeong JJ, Park SE, et al. Clinical and radiological outcomes of unstable proximal humeral fractures treated with a locking plate and fibular strut allograft. Int Orthop 2016;40:56977.

6. Tan E, Lie D, Wong MK. Early outcomes of proximal humerus fracture fixation with locking plate and intramedullary fibular strut graft. Orthopedics 2014;37:e822-7.

7. Zhao L, Qi YM, Yang L, et al. Comparison of the effects of Proximal Humeral Internal Locking System (PHILOS) alone and PHILOS combined with fibular allograft in the treatment of neer three- or four-part proximal humerus fractures in the elderly. Orthop Surg 2019;11:1003-12.

8. Liu K, Liu PC, Liu R, Wu X. Advantage of minimally invasive lateral approach relative to conventional deltopectoral approach for treatment of proximal humerus fractures. Med Sci Monit 2015;21:496-504.

9. Acklin YP, Jenni R, Walliser M, Sommer C. Minimal invasive PHILOS $\left({ }^{\circledR}\right)$-Pplate osteosynthesis in proximal humeral fractures. Eur J Trauma Emerg Surg 2009;35:35-9.

10. Roderer G, Erhardt J, Kuster M, et al. Second generation locked plating of proximal humerus fractures: a prospective multicentre observational study. Int Orthop 2011;35:425-32.

11. Sohn HS, Shin SJ. Minimally invasive plate osteosynthesis for proximal humeral fractures: clinical and radiologic outcomes according to fracture type. J Shoulder Elbow Surg 2014;23: 1334-40.

12. Palvanen M, Kannus P, Niemi S, Parkkari J. Update in the epide-
14. Gardner MJ, Griffith MH, Dines JS, Briggs SM, Weiland AJ, Lorich DG. The extended anterolateral acromial approach allows minimally invasive access to the proximal humerus. Clin Orthop Relat Res 2005;(434):123-9.

15. Sohn HS, Jeon YS, Lee J, Shin SJ. Clinical comparison between open plating and minimally invasive plate osteosynthesis for displaced proximal humeral fractures: a prospective randomized controlled trial. Injury 2017;48:1175-82.

16. Lin T, Xiao B, Ma X, Fu D, Yang S. Minimally invasive plate osteosynthesis with a locking compression plate is superior to open reduction and internal fixation in the management of the proximal humerus fractures. BMC Musculoskelet Disord 2014; 15:206.

17. Li F, Liu X, Wang F, et al. Comparison between minimally invasive plate osteosynthesis and open reduction-internal fixation for proximal humeral fractures: a meta-analysis based on 1050 individuals. BMC Musculoskelet Disord 2019;20:550.

18. Kim YG, Park KH, Kim JW, et al. Is minimally invasive plate osteosynthesis superior to open plating for fixation of two-part fracture of the proximal humerus. J Orthop Surg (Hong Kong) 2019;27:2309499019836156.

19. Bono CM, Grossman MG, Hochwald N, Tornetta P 3rd. Radial and axillary nerves: anatomic considerations for humeral fixation. Clin Orthop Relat Res 2000;(373):259-64.

20. Acklin YP, Stoffel K, Sommer C. A prospective analysis of the functional and radiological outcomes of minimally invasive plating in proximal humerus fractures. Injury 2013;44:456-60.

21. Laflamme GY, Rouleau DM, Berry GK, Beaumont PH, Reindl R, Harvey EJ. Percutaneous humeral plating of fractures of the proximal humerus: results of a prospective multicenter clinical trial. J Orthop Trauma 2008;22:153-8.

22. Gardner MJ, Boraiah S, Helfet DL, Lorich DG. Indirect medial reduction and strut support of proximal humerus fractures using an endosteal implant. J Orthop Trauma 2008;22:195-200.

23. Bae JH, Oh JK, Chon CS, Oh CW, Hwang JH, Yoon YC. The biomechanical performance of locking plate fixation with intramedullary fibular strut graft augmentation in the treatment of unstable fractures of the proximal humerus. J Bone Joint Surg Br 2011;93:937-41.

24. Matassi F, Angeloni R, Carulli C, et al. Locking plate and fibular allograft augmentation in unstable fractures of proximal hu- 
merus. Injury 2012;43:1939-42.

25. Cha H, Park KB, Oh S, Jeong J. Treatment of comminuted proximal humeral fractures using locking plate with strut allograft. J Shoulder Elbow Surg 2017;26:781-5.

26. Cui X, Chen H, Ma B, Fan W, Li H. Fibular strut allograft influences reduction and outcomes after locking plate fixation of comminuted proximal humeral fractures in elderly patients: a retrospective study. BMC Musculoskelet Disord 2019;20:511.

27. Noh YM, Kim DR, Kim CH, Lee SY. Minimally invasive percu- taneous plate osteosynthesis via a deltoidsplitting approach with strut allograft for the treatment of displaced 3- or 4-part proximal humeral fractures. Clin Should Elbow 2018;21:220-6.

28. Kim DS, Lee DH, Chun YM, Shin SJ. Which additional augmented fixation procedure decreases surgical failure after proximal humeral fracture with medial comminution: fibular allograft or inferomedial screws. J Shoulder Elbow Surg 2018;27: 1852-8. 